\title{
Combination of Osthole and Cisplatin Against Rhabdomyosarcoma TE671 Cells Yielded Additive Pharmacologic Interaction by Means of Isobolographic Analysis
}

\author{
AGATA JARZĄB ${ }^{1}$, JAROGNIEW ŁUSZCZKI ${ }^{2}$, MAŁGORZATA GUZ ${ }^{1}$, KRYSTYNA SKALICKA-WOŹNIAK ${ }^{3}$, \\ MARTA HAŁASA ${ }^{1}$, JOLANTA SMOK-KALWAT ${ }^{4}$, KRZYSZTOF POLBERG $^{5}$ and ANDRZEJ STEPULAK ${ }^{1}$ \\ ${ }^{1}$ Department of Biochemistry and Molecular Biology, Medical University of Lublin, Lublin, Poland; \\ ${ }^{2}$ Department of Pathophysiology, Medical University of Lublin, Lublin, Poland; \\ ${ }^{3}$ Department of Pharmacognosy with Medical Plant Unit, Medical University of Lublin, Lublin, Poland; \\ ${ }^{4}$ Department of Clinical Oncology, Holy Cross Cancer Centre, Kielce, Poland; \\ ${ }^{5}$ Department of Otolaryngology, MSWiA Hospital, Lublin, Poland
}

\begin{abstract}
Background/Aim: Osthole is a simple coumarin that has been found to have anticancer, anti-inflammatory, antiviral, anticoagulant, anticonvulsant and antiallergic activities. The aim of this study was to analyze the combined anti-proliferative effect of cisplatin $(C D D P)$ and osthole on a rhabdomyosarcoma cell line, and assess the pharmacology of drug-drug interaction between these drugs using isobolographic analysis. Materials and Methods: The anticancer actions of osthole in combination with CDDP were evaluated using the tetrazolium dye-based MTT cell proliferation assay. Results: Osthole and CDDP applied together augmented their anti-cancer activities and yielded an additive type of pharmacologic interaction by means of isobolographic analysis. Conclusion: Combined therapy using osthole and cisplatin could be suggested as a potential chemotherapy regimen against rhabdomyosarcoma.
\end{abstract}

Cis-diamminedichloroplatinum (II) (Cisplatin, CDDP) is a widely used chemotherapeutic agent in the treatment of various neoplasias. CDDP is considered the mainline treatment of cancers of the ovaries, testes, and solid tumors of the head and neck (1). Despite its toxic side-effects, including severe kidney problems, gastrointestinal disorders, hemorrhage, and hearing loss, CDDP is also used against

Correspondence to: Andrzej Stepulak, Department of Biochemistry and Molecular Biology, Medical University of Lublin, Lublin, Poland. Tel: +48 814486352, Fax: +48 814486350, e-mail: andrzej.stepulak@umlub.pl

Key Words: Osthole, isobolography, CDDP, rhabdomyosarcoma. cancers of soft tissue, bones (2), muscles, and sarcomas (3), including rhabdomyosarcoma (RMS) (4). RMS originating from mesenchymal stem cells develop malignant tumors of soft tissues located often in the head/neck and genitourinary system in children and adolescents causing aggressive disease and clinical complications (5). Patients diagnosed with RMS have a poor prognosis, which is caused by late diagnosis, metastasis and local recurrence (6). Most patients with RMS are treated by surgery, radiotherapy and chemotherapy consisting of vincristine, actinomycin and cyclophosphamide (VAC) as standard therapy. Although many other chemotherapy regimens (vincristine, topotecan and cyclophosphamide -VTC, etoposide, ifosfamide and vincristine - IEV, actinomycin. etoposide and vincristine DEV) have been applied, no significant statistical relationship between protocol of treatment and RMS outcome has yet been reported (7). Slightly better results were observed for protocols consisting of vincristine, cyclophosphamide and cisplatin (VCP) (4), suggesting that CDDP may augment RMS chemotherapy. Nevertheless, RMS treatment outcome has not been improved for three decades (5). Thereby, better novel approaches or additive treatments are needed.

In our previous study, we demonstrated that CDDP inhibits RMS cells (TE671 cell line) proliferation, especially when combined with histone deacetylase inhibitors (8). On the other hand, we also reported that simple coumarin osthole (7methoxy-8-(3-methyl-2-butenyl)-2H-1-benzopyran-2-one), a natural product derived from medicinal plants display anticancer activity against RMS TE671 cell line by inhibiting their proliferation, inducing apoptosis, and slowing down the cell cycle progression, presumably trough increase of TP53 and CDKNIA genes expression (9). Osthole has also been 
Table I. Anti-proliferative effects of CDDP and osthole (OST) administered singly in the TE671 cancer cell line.

\begin{tabular}{|c|c|c|c|c|c|c|}
\hline $\begin{array}{l}\text { Drug } \\
\text { Ratio S.R. }\end{array}$ & $\begin{array}{c}\mathrm{IC}_{50}(\mu \mathrm{g} / \mathrm{ml}) \\
\text { parallelism }\end{array}$ & $n$ & CFP & $q / p$ & S.R. & $f$ \\
\hline CDDP & $\begin{array}{c}0.591 \pm 0.187 \\
-\end{array}$ & 48 & $0.363(p)$ & - & - & - \\
\hline OST & $\begin{array}{c}4.049 \pm 1.053 \\
\text { NP }\end{array}$ & 60 & $0.682(q)$ & 1.879 & 4.645 & 3.557 \\
\hline
\end{tabular}

Results are presented as median inhibitory concentrations $\left(\mathrm{IC}_{50}\right.$ values in $\mu \mathrm{g} / \mathrm{ml} \pm$ S.E.M.) of CDDP and osthole (OST) administered singly with respect to their anti-proliferative effects in the cancer cell line (TE671) measured in vitro by the MTT assay. $n$ : Total number of items used at concentrations whose expected anti-proliferative effects ranged between 4 and 6 probits (16\% and 84\%); CFP: ( $q$ and $p$ ) curve-fitting parameters; $q / p$ : ratio of $q$ and $p$-values; S.R.: slope function ratio $\left(\mathrm{S}_{\mathrm{CDDP}} / \mathrm{S}_{\mathrm{OST}}\right) ; \mathrm{f}$ ratio. Test for parallelism of two DRRCs was performed according to Litchfield and Wilcoxon (1949) (19). It this case, if the slope function ratio (S.R.) value is higher than the factor for slope function ratio (f ratio S.R.) value, the examined two DRRCs are not parallel to each other (19). NP: Not parallel; *All detailed calculations required to test the parallelism of two DRRCs were presented in the Appendix to the paper by Luszczki and Czuczwar (2006) (25).

reported to inhibit migration and invasiveness of several other cancer cell lines, including breast cancer cells (10), lung adenocarcinoma (11), hepatocellular carcinoma (12), glioma (13), and osteosarcoma (14). In the present study, we combined osthole and CDDP in an experimental treatment against RMS cells in order to assess their pharmacological interaction by means of advanced isobolographic analysis.

\section{Materials and Methods}

Cell culture. TE671, human rhabdomyosarcoma cell line was obtained from the European Collection of Cell Cultures (ECACC). Mycoplasma free cells were routinely maintained in Dulbecco's modified Eagle's medium F12 Ham (Sigma - Aldrich, St. Louis, MO, USA) supplemented with $10 \% \mathrm{v} / \mathrm{v}$ fetal bovine serum and antibiotics penicillin $(100 \mathrm{~g} / \mathrm{ml})$ and streptomycin $(100 \mathrm{~g} / \mathrm{ml})($ Sigma - Aldrich, St. Louis, MO, USA) incubated at $37^{\circ} \mathrm{C}$ in humidified atmosphere containing $5 \% \mathrm{CO}_{2}$.

Cell treatments. Cisplatin (Sigma - Aldrich, St. Louis, MO, USA), and osthole were dissolved in phosphate buffered saline (PBS) with $\mathrm{Ca}^{2+}$ and $\mathrm{Mg}^{2+}$, or in methanol as stock solutions, respectively. Osthole (OST) was isolated from fruits of Mutellina purpurea using high-performance counter-current chromatography (HPCCC) as we described previously in detail (9). TE671 cells $\left(1 \times 10^{4}\right.$ cells/well $)$ were seeded into 96 -well cell culture plates for $24 \mathrm{~h}$ at $37^{\circ} \mathrm{C}$. Next day, cells were incubated with increasing concentrations of osthole $(0,405-4,86 \mu \mathrm{g} / \mathrm{ml})$ and cisplatin $(0,06-0,71 \mu \mathrm{g} / \mathrm{ml})$ for $96 \mathrm{~h}$.

MTT assay. Inhibition of cancer cells proliferation was evaluated by 3-(4,5-dimethylthiazol-2-yl)-2,5-diphenyltetra-zolium bromide
Table II. Type I isobolographic analysis of interaction between CDDP and OST at the fixed-ratio combination of 1:1 in the cancer cell line TE671 measured in vitro by the MTT assay.

\begin{tabular}{lccccc}
\hline $\begin{array}{l}\mathrm{IC}_{50} \mathrm{mix} \\
(\mu \mathrm{g} / \mathrm{ml})\end{array}$ & $n$ mix & $\begin{array}{c}\mathrm{a}_{\mathrm{IC}} \\
(\mu \mathrm{g} / \mathrm{ml})\end{array}$ & $n_{\text {add }}$ & $\begin{array}{c}\mathrm{b}^{\mathrm{II}} \text { 50 add } \\
(\mu \mathrm{g} / \mathrm{ml})\end{array}$ & $n_{\text {add }}$ \\
\hline $3.568 \pm 0.555$ & 120 & $1.820 \pm 0.943$ & 104 & $2.819 \pm 1.002$ & 104 \\
\hline
\end{tabular}

Results are presented as median inhibitory concentrations $\left(\mathrm{IC}_{50}\right.$ values in $\mu \mathrm{g} / \mathrm{ml} \pm$ S.E.M.) for the two-drug mixture, determined either experimentally $\left(\mathrm{IC}_{50 \mathrm{mix}}\right)$ or theoretically calculated $\left(\mathrm{IC}_{50}\right.$ add $)$ from the equations of additivity, blocking proliferation in $50 \%$ of tested cells in the cancer cell line (TE671) measured in vitro by the MTT assay. $\mathrm{n}$ mix - total number of items used at those concentrations whose expected anti-proliferative effects ranged between $16 \%$ and $84 \%$ (i.e., 4 and 6 probits) for the experimental mixture; $n_{\text {add }}$ : Total number of items calculated for the additive mixture of the drugs examined $\left(n_{\text {add }}=n\right.$ CDDP $\left.+n_{\text {OST }}-4\right) ;{ }^{\text {a }} \mathrm{C}_{50}$ add value calculated from the equation for the lower line of additivity; ${ }^{{ } \mathrm{IC}_{50}}$ add value calculated from the equation for the upper line of additivity. Statistical evaluation of data was performed with unpaired Student's $t$-test.

(MTT) assay as we described previously (8). After treatment with the examined compounds the cells were incubated with $10 \mu \mathrm{l}$ of MTT solution ( $5 \mathrm{mg} / \mathrm{ml}$, Sigma - Aldrich, St. Louis, MO, USA). The reaction was stopped after 3 using $10 \%$ SDS in $0,01 \mathrm{~N} \mathrm{HCl}$ solution. Finally, absorbance was measured at $570 \mathrm{~nm}$ (Infinite M200 Pro Microplate Reader, Tecan, Männedorf, Switzerland).

Isobolographic analysis. Isobolographic analysis- a statistical method allowing the precise characterization of pharmacodynamic interaction between drugs (15) was performed as described previously $(8,16-18)$, First, the percent inhibition of cell viability per increasing doses of CDDP, and osthole administrated singly in the rhabdomyosarcoma cell line TE671 was measured. Subsequently, the dose-response effects for each investigated anticancer compound (i.e., CDDP, osthole) were fitted with log-probit linear regression analysis as described by Litchfield and Wilcoxon (1949) (19). The test for parallelism of dose-response effects for CDDP and osthole, revealed that CDDP had its dose-response effect non-parallel to that of osthole in the rhabdomyosarcoma cell line (TE671) measured by the MTT assay. The type of interactions between CDDP and osthole in the cancer cell line TE671 was isobolographically analyzed according to the methodology described elsewhere $(16,20)$. From the experimentally denoted $\mathrm{IC}_{50}$ values for the drugs administered alone, median additive inhibitory concentrations of the mixture of CDDP with osthole at the fixedratio of 1:1 $\left(\mathrm{IC}_{50 \text { add }}\right)$ - i.e., concentrations of the mixture, which should theoretically inhibit cell viability in $50 \%$ were calculated as described earlier (16). Subsequently, the experimentally-derived $\mathrm{IC}_{50 \mathrm{mix}}$ at the fixed-ratio of 1:1 was determined based on the concentration of the mixtures of CDDP with osthole, inhibiting 50\% of cell viability in the cancer cell line (TE671) measured in vitro by the MTT assay. The separate concentrations of CDDP and osthole in the mixture were calculated from, the $\mathrm{IC}_{50 \mathrm{mix}}$ values by multiplying this value by the respective proportions of particular drugs. Additional information concerning the isobolographic analysis has been published elsewhere $(16,20)$. 


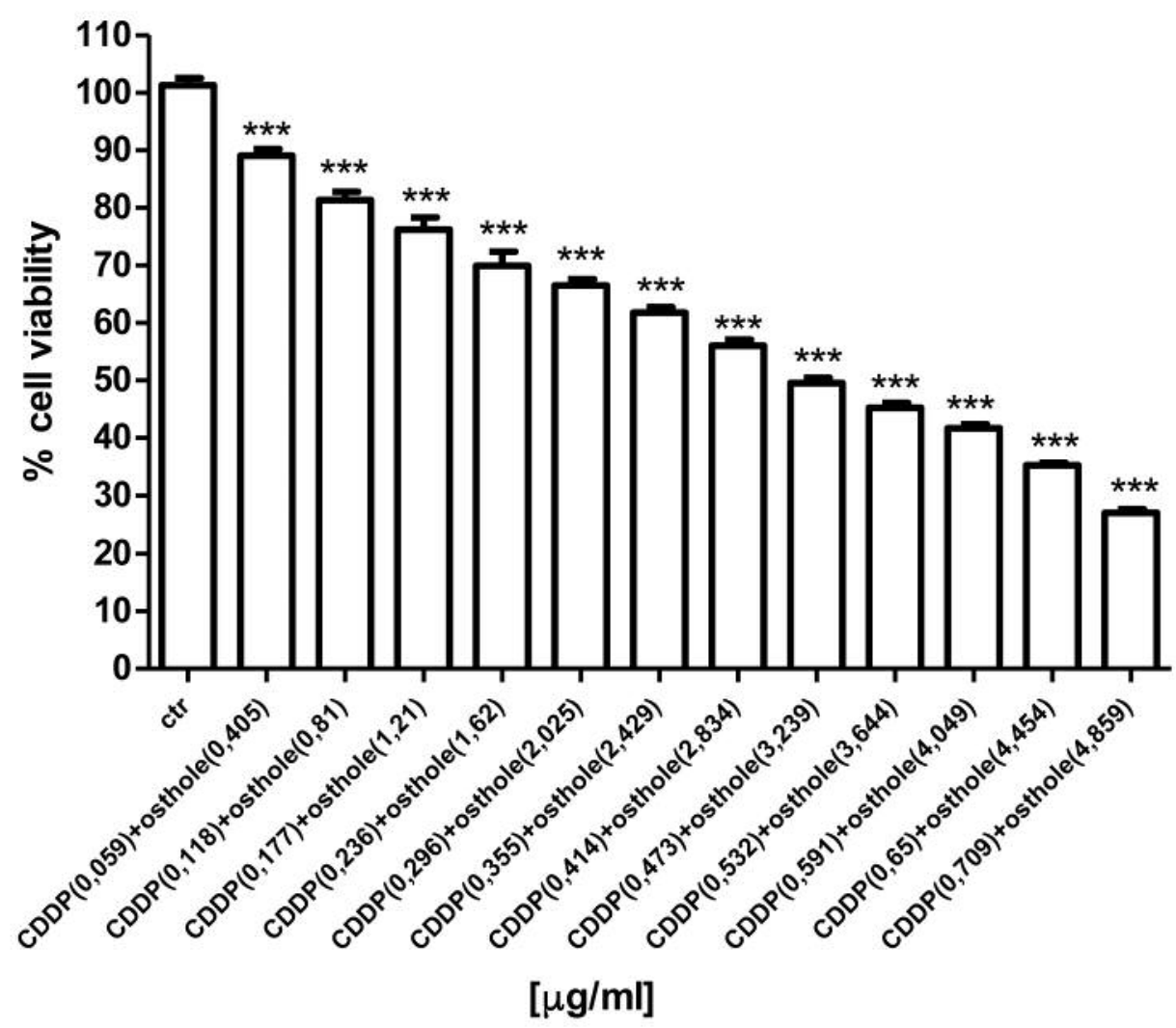

Figure 1. TE671 cells were incubated with 1:1 drug mixture (CDDP/osthole) in increasing concentrations for 96 h showing a dose-dependent decrease of cancer cell viability (MTT test). Each bar represents mean optical densities $\pm S . E . M .,{ }^{* * *} p<0.001$ versus control; Student's test) of 24 measurements from three independent experiments.

Statistical analysis. GraphPad Prism software was used for data analyzing (One-way ANOVA with Tukey post hoc test). $p<0.05$ was considered to indicate a statistically significant difference. Results were presented as mean \pm standard error of the mean (SEM).

Log-probit analysis was used to determine the experimentallyderived $\mathrm{IC}_{50}$ and $\mathrm{IC}_{50 \text { mix }}$ values for CDDP, and osthole, when the drugs were administered alone or in combination for the fixed-ratio of 1:1 (19). Difference between the experimentally-derived $\mathrm{IC}_{50 \text { mix }}$ values for the mixture of CDDP with osthole and the theoretically additive $\mathrm{IC}_{50}$ add values was statistically verified by using the unpaired Student's $t$-test, as presented elsewhere (20).

\section{Results}

As we demonstrated previously, CDDP (8) and osthole (9) inhibited TE671 cells proliferation in a dose-dependent manner when applied separately. In the present study using Log-probit dose-response analysis we determined $\mathrm{IC}_{50}$ for osthole (Table I), whereas $\mathrm{IC}_{50}$ for CDDP is $0.591 \pm 0.187$, as we demonstrated previously (8). Next, we evaluated the antiproliferative effects of osthole administered in combination with CDDP to the TE671 cell line. Incubation of RMS cells with different concentrations of both drugs based on established $\mathrm{IC}_{50}$ values resulted in dose-dependent reduction of cancer cells viability (Figure 1).

The test for parallelism of dose-response effects between CDDP and osthole confirmed that the log-probit lines of these compounds were non-parallel to one another (Table I; Figure 2).

The combinations of CDDP with osthole (at the fixed-ratio of 1:1) produced the definite anti-proliferative effects in the TE671 cell line and the experimentally-determined $\mathrm{IC}_{50 \mathrm{mix}}$ values for the two-drug mixture were $3.568 \pm 0.555 \mu \mathrm{g} / \mathrm{ml}$ for the combination of CDDP with osthole (Table II, Figure 3). With type I isobolographic analysis, no statistical difference was observed between the $\mathrm{IC}_{50 \text { mix }}$ and $\mathrm{IC}_{50}$ add values with unpaired Student's $t$-test and thus, the analyzed interactions between CDDP and osthole were additive (Table II).

\section{Discussion}

Chemotherapy of rhabdomyosarcoma remains an oncological challenge. VAC (vincristin, adriamycin plus cyclophosphamide) has been considered the gold standard regimen for RMS. A 


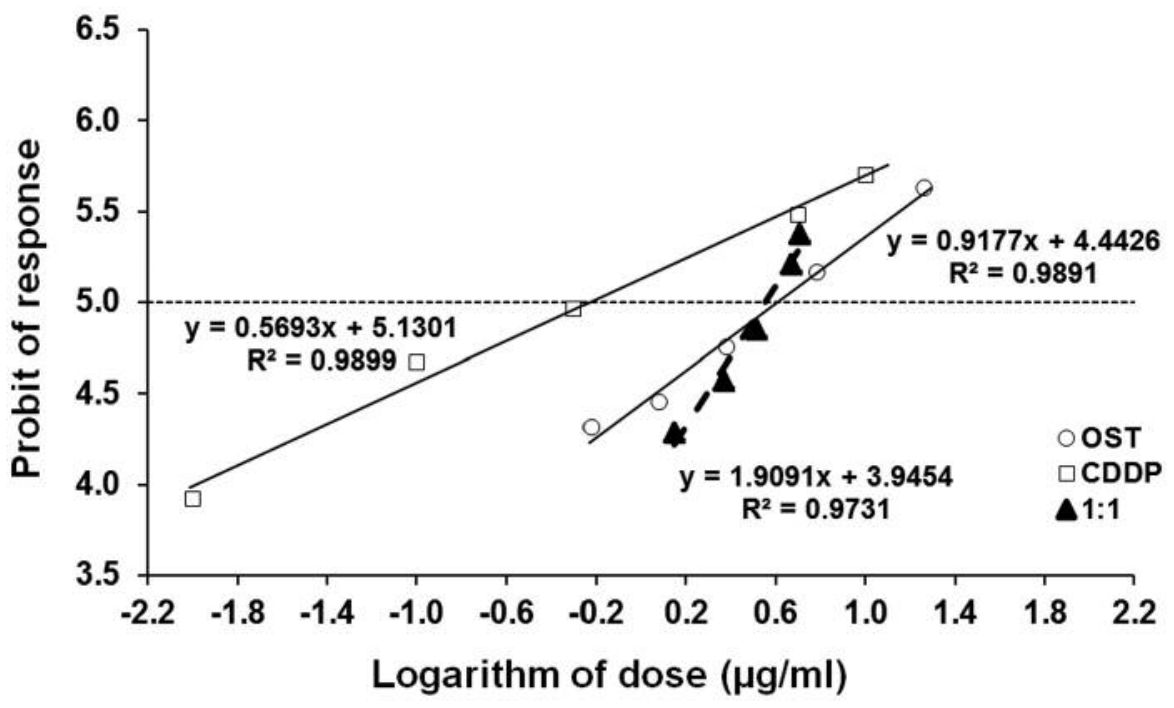

Figure 2. Log-probit dose-response relationship curves (DRRCs) for cisplatin (CDDP) and osthole (OST) administered alone, and in combination at the fixed-ratio of 1:1 (in red), illustrating the anti-proliferative effects of the drugs in the cancer cell line (TE671) measured in vitro by the MTT assay. Doses of CDDP and OST administered separately and the mixture of the drugs at the fixed-ratio combination of 1:1 (in red) were transformed into logarithms, whereas the anti-proliferative effects produced by the drugs in the cancer cell line (TE671) measured in vitro by the MTT assay were transformed into probits according to Litchfield and Wilcoxon (1949) (19). Linear regression equations of DRRCs are presented on the graph; where $y:$ is the probit of response, and $x$ : is the logarithm (to the base 10) of a drug dose, $R^{2}$ : coefficient of determination. Test for parallelism revealed that the experimentally determined DRRCs for CDDP and OST (administered alone) are not parallel to one another (for more details see Table I).

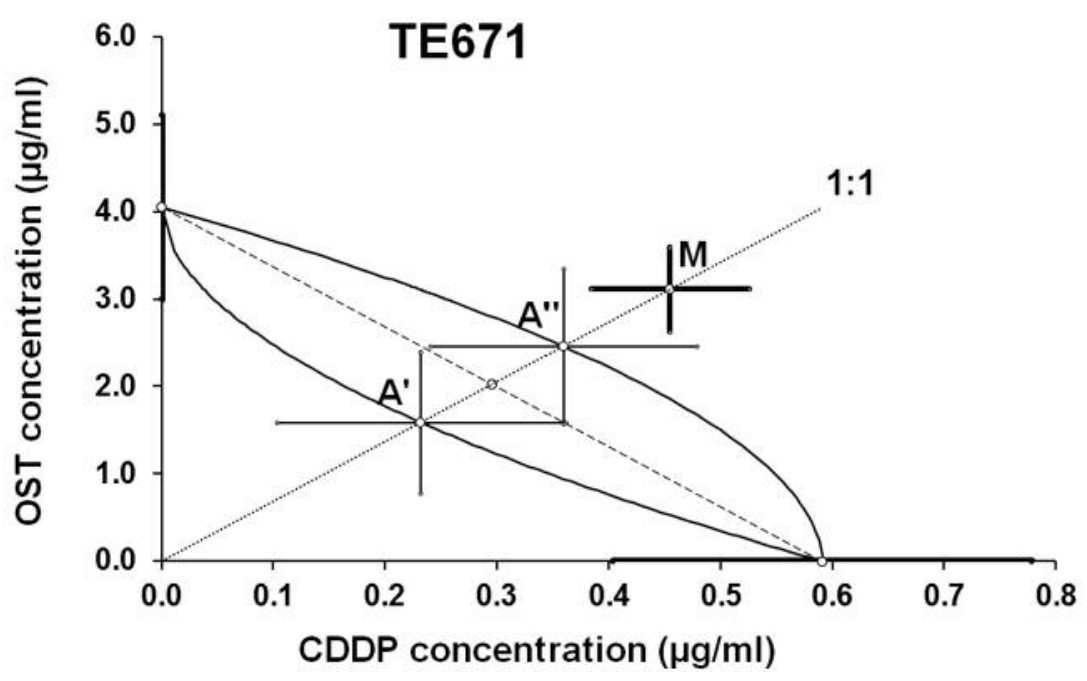

Figure 3. Isobologram showing additive interaction between cisplatin (CDDP) and osthole (OST) with respect to their anti-proliferative effects in the cancer cell line TE671, measured in vitro by the MTT assay. The median inhibitory concentrations $\left(I_{50}\right)$ for CDDP and OST are plotted on the $X$ - and $Y$-axes, respectively. The solid lines on the $X$ and $Y$ axes represent the S.E.M. for the $I_{50}$ values for the studied drugs administered alone. The lower and upper isoboles of additivity represent the curves connecting the $I C_{50}$ values for CDDP and OST administered alone. The dotted line starting from the point $(0,0)$ corresponds to the fixed-ratio of 1:1 for the combination of CDDP with OST. The diagonal dashed line connects the $I C_{50}$ for CDDP and OST on the X-and Y-axes. The points A' and A" depict the theoretically calculated IC 50 add values for both, lower and upper isoboles of additivity. The point $M$ represents the experimentally-derived IC50 mix value for total dose of the mixture expressed as proportions of CDDP and OST that produced a 50\% anti-proliferative effect in TE671 as measured in vitro by the MTT assay. On the graph, the S.E.M. values are presented as horizontal and vertical error bars for every $I C_{50}$ value. Although the experimentally-derived $I C_{50}$ mix value is placed above the point A", statistical analysis of data revealed no significant differences among $I C_{50}$ mix and $I_{50}$ add values. Thus, additive interaction exists between CDDP and OST in the cancer cell line TE671, as measured in vitro by the MTT assay. 
treating regime using many individually active agents (e.g. doxorubicin, cisplatin, etoposide, ifosfamide, topotecan, melphalan) failed to improve significantly outcomes as compared to VAC (4). Thereby, novel approaches or combinations of drugs that result in an additive treatment are needed.

Osthole has reportedly shown anticancer activity against several cancer cell types both in vitro $(10,11,21)$ and in vivo (12) including rhabdomyosarcoma cells (9). Osthole's mechanism of action has been attributed to the inhibition of cell cycle progression, induction of apoptosis (9) interfering with cancer survival signaling pathways, including AKT and ERK kinases, as well as attenuating matrix metalloproteinases activity (21). On the other hand, osthole displays several protective properties, including osteogenic and immuno-modulatory activities, as well as hepatoprotective and neuroprotective actions (21). In the context of anticancer therapy, causing often severe toxic side effects, the simultaneous use of osthole and CDDP seems to have a beneficial influence potentially ameliorating some conventional drug-induced side-effects. However, this issue requires further in vivo studies.

CDDP, an old drug, has recently received renewed attention, especially in the treatment of heterogeneous, rapidly proliferating cancer types, including triple-negative (lacking estrogen receptor, progesterone receptor, and HER expression) breast cancer cells (TNBC) (22). RMS cells are characterized by very fast, uncontrolled proliferation, and poor outcome for RMS-bearing patients (5). Thereby, in the present work we performed an experimental treatment of RMS cells combining osthole and CDDP in vitro. Using isobolographic analysis for the precise assessment of pharmacological drug-drug interaction type utilizing several combinations of both drugs we were able to show additive interaction of osthole and CDDP, resulting in a greatly enhanced anticancer effect of these drug combination compared to single drug treatment. Similar results we presented also recently combining CDDP and histone deacetylase inhibitors against RMS cells (8). This kind of combination therapy could be very promising in overcoming cisplatin resistance. Additionally, application of osthole decreased the doses of cisplatin used to achieve the same treatment efficiency and potentially ameliorating some of the CDDP-induced side effects. Combination therapy of osthole (23) or its derivative (24) and CDDP was also reported in other single reports, however usually very limited number of drug concentrations were examined, sometimes only one (23), which does not allow to establish a drug-drug type of pharmacokinetic interaction. In our present detailed study, using several $\mathrm{CDDP} /$ osthole combinations we were able to clearly demonstrate an additive interaction in RMS cells.

Beneficial effects of CDDP/osthole treatment have also been observed against NCI-H460 lung cancer cells in vitro (23), showing that this combination could be regarded as a general phenomenon and rather not cancer cell type-specific.
This could support the potential role of osthole as a drug candidate in cancer treatment.

\section{References}

1 Dasari S and Bernard Tchounwou P: Cisplatin in cancer therapy: Molecular mechanisms of action. Eur J Pharmacol 740: 364-378, 2014.

2 Wang $\mathrm{X}$, Zheng $\mathrm{H}$, Shou $\mathrm{T}$, Tang $\mathrm{C}$, Miao $\mathrm{K}$ and Wang P: Effectiveness of multi-drug regimen chemotherapy treatment in osteosarcoma patients: a network meta-analysis of randomized controlled trials. J Orthop Surg Res 12: 52, 2017.

3 Harrison DJ and Schwartz CL: Osteogenic sarcoma: Systemic chemotherapy options for localized disease. Curr Treat Options Oncol 18: 24, 2017.

4 Ma X, Huang D, Zhao W, Sun L, Xiong H, Zhang Y, Jin M and Zhang D: Clinical characteristics and prognosis of childhood rhabdomyosarcoma: a ten-year retrospective multicenter study. Int J Clin Exp Med 8: 17196-17205, 2015.

5 Kashi VP, Hatley ME and Galindo RL: Probing for a deeper understanding of rhabdomyosarcoma: insights from complementary model systems. Nat Rev Cancer 15: 426-439, 2015.

6 Yang L, Takimoto T and Fujimoto J: Prognostic model for predicting overall survival in children and adolescents with rhabdomyosarcoma. BMC Cancer 14: 654, 2014.

7 Hosoi H: Current status of treatment for pediatric rhabdomyosarcoma in the USA and Japan. Pediatr Int 58: 81-87, 2016.

8 Jarząb A, Łuszczki JJ, Guz M, Gumbarewicz E, Polberg K and Stepulak A: Additive interaction of cisplatin and histone deacetylase inhibitors combined treatment in rhabdomyosarcoma cells? An isobolographic analysis. Anticancer Res 37: 1067-1074, 2017.

9 Jarząb A, Grabarska A, Kiełbus M, Jeleniewicz W, DmoszyńskaGraniczka M, Skalicka-Woźniak K, Sieniawska E, Polberg K and Stepulak A: Osthole induces apoptosis, suppresses cell-cycle progression and proliferation of cancer cells. Anticancer Res 34: 6473-6480, 2014.

10 Yang D, Gu T, Wang T, Tang Q and Ma C: Effects of osthole on migration and invasion in breast cancer cells. Biosci Biotechnol Biochem 74: 1430-1434, 2010.

$11 \mathrm{Xu} \mathrm{X}$, Zhang Y, Qu D, Jiang T and Li S: Osthole induces $\mathrm{G}_{2} / \mathrm{M}$ arrest and apoptosis in lung cancer A549 cells by modulating PI3K/Akt pathway. J Exp Clin Cancer Res 30: 33, 2011.

12 Zhang L, Jiang G, Yao F, He Y, Liang G, Zhang Y, Hu B, Wu Y, $\mathrm{Li} \mathrm{Y}$ and Liu H: Growth inhibition and apoptosis induced by osthole, a natural coumarin, in Hepatocellular Carcinoma. PLoS One 7: e37865, 2012.

13 Ding D, Wei S, Song Y, Li L, Du G, Zhan H and Cao Y: Osthole exhibits anti-cancer property in rat glioma cells through inhibiting PI3K/Akt and MAPK signaling pathways. Cell Physiol Biochem 32: 1751-1760, 2013.

14 Ding Y, Lu X, Hu X, Ma J and Ding H: Osthole inhibits proliferation and induces apoptosis in human osteosarcoma cells. Int J Clin Pharmacol Ther 52: 112-117, 2014.

15 Gessner PK: Isobolographic analysis of interactions: an update on applications and utility. Toxicology 105: 161-179, 1995.

16 Luszczki JJ: Isobolographic analysis of interaction between drugs with nonparallel dose-response relationship curves: A practical application. Naunyn Schmiedebergs Arch Pharmacol 375: 105-114, 2007. 
17 Wawruszak A, Luszczki JJ, Grabarska A, Gumbarewicz E, Dmoszynska-Graniczka M, Polberg $\mathrm{K}$ and Stepulak A: Assessment of interactions between cisplatin and two histone deacetylase inhibitors in MCF7, T47D and MDA-MB-231 human breast cancer cell lines - An isobolographic analysis. PLoS One 10: e0143013, 2015.

18 Luszczki JJ, Filip D and Czuczwar SJ: Additive interactions of pregabalin with lamotrigine, oxcarbazepine and topiramate in the mouse maximal electroshock-induced seizure model: A type I isobolographic analysis for non-parallel dose-response relationship curves. Epilepsy Res 91: 166-175, 2010.

19 Litchfield JTJ and Wilcoxon F: A simplified method of evaluating dose-effect experiments. J Pharmacol Exp Ther 96: 99-113, 1949.

20 Tallarida RJ: Drug synergism: its detection and applications. J Pharmacol Exp Ther 298: 865-872, 2001.

21 Zhang Z-R, Leung WN, Cheung HY and Chan CW: Osthole: A review on its bioactivities, pharmacological properties, and potential as alternative medicine. evidence-based complement Altern Med 2015: 1-10, 2015.

22 Isakoff SJ, Mayer EL, He L, Traina TA, Carey LA, Krag KJ, Rugo HS, Liu MC, Stearns V, Come SE, Timms KM, Hartman AR, Borger DR, Finkelstein DM, Garber JE, Ryan PD, Winer EP, Goss PE and Ellisen LW: TBCRC009: A multicenter phase II clinical trial of platinum monotherapy with biomarker assessment in metastatic triple-negative breast cancer. J Clin Oncol 33: 1902-1909, 2015.
23 Xu XM, Zhang Y, Qu D, Liu HB, Gu X, Jiao GY and Zhao L: Combined anticancer activity of osthole and cisplatin in NCIH460 lung cancer cells in vitro. Exp Ther Med 5: 707-710, 2013.

24 Chen TJ, Zhou YF, Ning JJ, Yang T, Ren H, Li Y, Zhang S and Chen MW: NBM-T-BMX-OS01, an osthole derivative, sensitizes human lung cancer A549 cells to cisplatin through AMPK-dependent inhibition of ERK and Akt pathway. Cell Physiol Biochem 36: 893-906, 2015.

25 Łuszczki JJ and Czuczwar SJ: Gabapentin synergistically interacts with topiramate in the mouse maximal electroshock seizure model: An isobolographic analysis. Pharmacol Rep 58: 944-954, 2006.
Received October 19, 2017

Revised October 31, 2017

Accepted November 1, 2017 